\title{
The professional and personal characteristics of effective psychotherapists : a systematic review
}

\section{Heinonen, Erkki}

2020-05-18

Heinonen , E \& Nissen-Lie , H A 2020 , ' The professional and personal characteristics of effective psychotherapists : a systematic review ', Psychotherapy Research , vol. 30 , no. 4 , pp. 417-432 . https://doi.org/10.1080/10503307.2019.1620366

http://hdl.handle.net/10138/315679

https://doi.org/10.1080/10503307.2019.1620366

acceptedVersion

Downloaded from Helda, University of Helsinki institutional repository.

This is an electronic reprint of the original article.

This reprint may differ from the original in pagination and typographic detail.

Please cite the original version. 
The professional and personal characteristics of effective psychotherapists: a systematic review

\author{
Erkki Heinonen $^{\mathrm{a}, \mathrm{b}}$, Helene A. Nissen-Lie ${ }^{\mathrm{c}}$

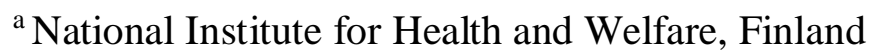 \\ ${ }^{\mathrm{b}}$ University of Helsinki, Finland \\ ${ }^{\mathrm{c}}$ University of Oslo, Norway
}

Address for correspondence: Erkki Heinonen, National Institute for Health and Welfare

Mannerheimintie 166b, P.O. Box 30, FIN-00271 Helsinki, Finland. Tel.: +358 29524 8774. Fax:

+35829524 8760. Email: erkki.heinonen@thl.fi

Acknowledgements: We are grateful to Professor David E. Orlinsky for his extremely valuable comments to the final draft of this manuscript. The first author gratefully acknowledges the support of the Finnish Cultural Foundation. 


\begin{abstract}
Objective: Psychotherapists differ notably in the outcomes their patients achieve, and the characteristics that may explain these differences have attracted increasing interest. We systematically review studies on therapist pre-treatment characteristics predicting patient outcomes.

Method: Systematic searches on databases for psychotherapy research, clinical psychology, and medical science for the years 2000-2018 identified published research examining therapist characteristics and psychotherapy outcomes. Of 2041 studies, 31 met inclusion criteria. Results: Findings show a few direct effects of therapist intrapersonal variables (e.g., self-relatedness, attachment) and several interaction effects with other constructs (e.g., patient pathology) on outcome. There is little support for the relevance of self-rated social skills. However, more consistent evidence has recently emerged for performance-based measurements of professional interpersonal skills, especially when elicited in challenging situations. Patient outcomes were also predicted by therapists' self-rated professional characteristics, such as their experienced difficulties in practice, coping mechanisms, and attitudes towards therapeutic work, indicating that therapist self-perception also matters, although not always in the direction expected. Conclusions: More effective therapists seem characterized by professionally cultivated interpersonal capacities, which are likely rooted in their personal lives and attachment history. Research guidelines are proposed for moving this field forward (including larger samples, multilevel modeling, and in-depth qualitative work).
\end{abstract}

Keywords: therapist characteristics; therapist effects; outcome; systematic review.

\title{
Introduction
}

As one avenue to better understand the considerable variation in therapeutic outcomes between patients (Lambert, 2013), several researchers have turned attention to the person delivering the psychotherapy, i.e., the psychotherapist (Castonguay \& Hill, 2017). Recent studies convincingly establish that some therapists achieve better outcomes than others. Further, the effects in these 
analyses are not explained by variation in the patients seen, by dyadic effects (i.e., the specific combination of patient and therapist), or by random error (Wampold, Baldwin, Grosse Holtforth, \& Imel, 2017). These 'therapist effects' might be expected to be stronger in naturalistic settings. However, they have also been observed with manualized treatments and in randomized controlled trials (Baldwin \& Imel, 2013; Johns, Barkham, Kellett, \& Saxon, 2019).

Several studies have shown stability of therapist effects across patient characteristics (such as age and diagnosis) as well as time and outcome domains (Goldberg, Rousmaniere et al., 2016; Nissen-Lie et al., 2016; Wampold \& Brown, 2005). For example, more effective therapists at a given time are more helpful also when measured later across subsequent cases (Wampold \& Brown, 2005). Similarly, therapists who are effective or ineffective in treating one problem type have also been shown to be more effective or ineffective with another problem type (Nissen-Lie et al., 2016). However, other studies indicate that therapists may also have particular problem-specific expertise (Kraus et al., 2016). In any case, there appears reason to believe that even if therapists are affected by patient characteristics (such as type of mental health problem, mood, or interpersonal style), the more effective therapists bring something to their work which is independent of the individual client they see.

A pressing task is therefore to understand the more or less stable characteristics of therapists that explain their differences in outcome. Besides helping to better understand how psychotherapy works, knowledge on the characteristics of effective therapists would have much other practical value. First, some characteristics may be dispositional, innate, or developed over a long period of time, and might be useful as criteria for selecting candidates to therapist training. Second, insofar as these qualities are trainable and modifiable, training programs and supervision could be geared to nurture these qualities. Third, merely being aware of these beneficial characteristics might help therapists monitor themselves in delivering the qualities shown to improve outcomes via reflective and deliberate practice (Goldberg, Babins-Wagner et al., 2016). Toward these aims, the present study 
reviews and evaluates the recent work on psychotherapist characteristics that affect therapy outcomes.

Earlier literature in this area was comprehensively reviewed in two volumes (i.e., the $4^{\text {th }}$ and $5^{\text {th }}$ editions) of the Handbook of Psychotherapy and Behavior Change (Beutler, Machado, \& Neufeldt, 1994; Beutler et al., 2004). However, the latest $6^{\text {th }}$ edition did not update knowledge on discrete therapist variables impacting outcomes but instead offered a meta-analysis of therapist effects (Baldwin \& Imel, 2013). More recently, Lingiardi and colleagues (2018) reviewed studies of how therapist characteristics predict outcomes in psychodynamic therapies. However, a systematic comprehensive review of studies published after 2000 and across a variety of therapeutic approaches is still due, which the present review delivers, and suggests promising avenues for future research.

To focus the review, several choices were made. First, defining the range of therapist characteristics to investigate was informed by the taxonomy of the previous reviews that classified therapist variables along two axes: Objective vs. Subjective and Therapy-Specific vs. CrossSituational (Beutler et al., 1994, 2004). In this scheme, objective characteristics can be relatively readily observed or verified through collateral reports (e.g., age, sex, profession, level of experience). Subjective qualities are hypothetical constructs (e.g., personality, values) whose identification relies more on inference (e.g., via self-report measures or observation). Therapy-specific characteristics (e.g., therapeutic philosophy, relational skills specific to working with clients) have been developed as part of the therapist role. Cross-situational characteristics describe therapists also in their nonprofessional and personal lives (e.g., cultural orientation, attachment style, personal values). For simplicity, we will refer to therapists' therapy-specific characteristics as professional and crosssituational qualities as personal characteristics.

Prior reviews have concluded that the objective characteristics of therapists—such as profession, gender, or competence and adherence to a treatment manual—are of limited value and do not consistently distinguish more from less effective therapists (e.g., Beutler et al., 2003; Crits- 
Christoph, Baranackie, Kurcias, \& Beck, 1991; Webb, DeRubeis, \& Barber, 2010). We therefore focused our review on the subjective (or inferred) professional and personal qualities of the clinician, which prior studies suggest as more promising in explaining therapist effects (Beutler et al., 2004). In many cases, these characteristics may also have practical implications, since they may more readily be modified through training or supervision than objective characteristics like background profession, age, or years of experience.

Second, a methodological criterion was used to focus our review on therapist "pre-treatment" or "input" variables (Orlinsky, Rønnestad, \& Willutzki, 2004); that is, on characteristics measurable prior to and irrespective of engagement with particular patients. The aim here was to identify the skills, traits and qualities that more effective therapists initially bring to each of their therapy sessions independent of the interaction with particular patients. By focusing our review in these ways, we hope to gain a clearer understanding of what therapists bring to their work that contributes to psychotherapeutic change. Our review starts with studies published from 2000, when Beutler et al. (2004) ended theirs, onwards through 2018.

\section{Rationale and study aims}

The current review synthesizes the literature on therapist characteristics that affect final or 'distal' therapy outcomes of patients - i.e., functioning and well-being assessed at the end of treatment or further follow-up (Hayes, Gelso, Goldberg, \& Kivlighan, 2018) - to get a clearer understanding of the qualities underlying the well-documented between-therapist differences in these outcomes (Johns et al., 2019). Studies focusing solely on intermediate or session outcomes (i.e., proximate outcomes) are thus not included. Based on the previous research mentioned, the therapist characteristics of interest had to be inferred (i.e., psychological constructs) rather than "objective" (e.g., age, sex, profession) (Beutler et al., 2004). Even if it was not the primary focus of the review, we noted potential moderators of therapist factors and treatment outcome, such as therapy type and length as well as certain patient characteristics (e.g., problem type, psychological qualities) that might help 
understand divergent results and add more nuance to the findings. Across treatments and patients, our goal was to identify similarities and differences in therapist qualities conducive to better outcomes. Finally, the review addresses the implications of the findings for training, practice, and further research.

\section{Method}

\section{Study Selection}

Relevant studies were identified by searching relevant databases for articles published between January 2000 through December 2018, including PsycInfo, MEDLINE, Scopus and Web of Science. Search terms are presented in Appendix A. Inclusion and exclusion criteria are presented in Appendix B.

Figure 1 illustrates the selection process through the different phases. The initial database search produced 3,027 records in total. The reference manager software RefWorks was used to remove duplicates, leading to 2,034 records for screening. Examination of review articles and chapters, checking reference lists, and consulting with colleagues identified another seven publications for potential inclusion. A total of 2,041 records were screened based on titles and abstracts, of which 1,923 were excluded for not meeting inclusion criteria. The reasons for exclusion included focus on somatic issues or disorders (HIV, cancer, stroke, genetic counseling, etc.); theoretical, clinical, or review studies; or lack of pre-treatment therapist and/or distal outcome measure (cf. Appendix B). The remaining 118 studies were retrieved in full and examined further. All searches revealed that no similar systematic review had been published since Beutler et al.'s (2004) chapter. Finally, a total of 31 articles were deemed suitable for inclusion.

\section{Data Synthesis}

The studies were notably heterogeneous: Most importantly, the therapist measures and constructs studied were quite diverse. Secondly, the statistical methods, the reporting of results, and the outcome measures also varied considerably. Consequently, a quantitative meta-analysis was not 
feasible, and a narrative approach was instead used to synthesize the study findings. This involved describing, critically appraising, and comparing the reviewed studies, plus integrating the findings into a narrative within a coherent theoretical framework.

\section{Results}

\section{Descriptive Characteristics of the Studies}

\section{Settings and design.}

Supplement Table 1 summarizes the descriptive characteristics of the reviewed studies. The 31 studies were collected from 21 separate practice settings. These comprised seven primarily public health settings (community mental health centers, outpatient clinics, and hospitals), fourteen university clinics (typically serving also community health care), and two private practice settings. The study designs varied from trials in which patients were randomized into treatment conditions or therapists into receiving supervision or outcome feedback (or not) to observational naturalistic studies. All studies consisted of longitudinal, prospectively collected patient outcome data. In two studies, therapists' agreement to participate and data on their characteristics were collected after the patient outcome data were almost (Anderson, Ogles, Patterson, Lambert, \& Vermeersch, 2009) or fully (Pereira, Barkham, Kellett, \& Saxon, 2017) collected.

\section{Patient characteristics.}

The number of patients varied greatly between studies (range 18 -4980). With the exception of one study investigating children treated in a group setting (Muratori et al., 2017), all involved adult patients in individual therapy. Depression was the most common disorder in 16 of the 20 studies reporting specific diagnoses, anxiety the most common in three studies. The proportion of Axis II personality disorders, when reported, varied from 3.3\% (Odyniec et al., 2017) to 84.7\% (Bruck et al., 2006).

\section{Treatment characteristics.}


Therapy orientation was sometimes reported as a treatment condition (e.g.,as a study characteristic) and sometimes as a treatment provider characteristic. The former was the natural case for those 14 studies whose primary design aimed to evaluate or compare distinct treatments. Most of the remaining 17 studies reported the theoretical orientation endorsed by therapists, sometimes as a mutually exclusive choice between different orientations (e.g., Pereira et al., 2017) and other times allowing therapists to endorse multiple frameworks and reporting the most salient theoretical influences (e.g., Nissen-Lie et al., 2017). The theoretical frameworks of treatments comprised all the major psychotherapeutic approaches. The reported mean number of therapy sessions ranged from 4 (Pereira et al., 2017) to 642 sessions (Sandell et al., 2000). The reported therapy duration ranged from 6 weeks (Dinger et al., 2007) to 5 years (Heinonen et al., 2014). The most commonly reported session frequency was approximately one session per week.

\section{Therapist characteristics.}

Therapists were most often psychologists by profession. However, psychiatrists, psychiatric nurses, and social workers were also represented in many studies. Clinical experience ranged from less than 1 year for participants in some studies (de Jong et al., 2012; Dinger et al., 2007; Pereira et al., 2017) to a mean of 21 years in others (Sandell et al., 2000). In addition, 12 of the 26 studies used graduate students as therapists, who typically had at least a master's level training or were currently in a doctoral program, and had varying amounts of clinical experience. The majority of the therapists were female, except in four studies (Anderson et al., 2009; Dinger et al., 2007; Hilliard, Henry, \& Strupp, 2000; Schauenburg et al., 2010). The therapists' mean age, when reported, ranged between 24 and 54 years across studies. Most studies did not report the therapists' ethnicity. In those that did, the vast majority was "White/Caucasian."

\section{Measures}

\section{Therapists' professional and personal characteristics.}


Therapist characteristics used as predictors were assessed both with self-ratings and observerrated measures (see Supplement Table 2 for all measured used). Some of the instruments were developed specifically for evaluating professional therapists. Other instruments used were developed for assessing broader populations. Their scope ranged from resources and skills, such as mindfulness, resilience, and social skills, to factors reflecting potential strengths as well as vulnerabilities, such as neuroticism, introject status, early parental care, attachment or interpersonal problems. With one exception, a categorical interview measure (Schauenburg et al., 2010), all self- and observer-rated characteristics studied as predictors were based on averaged multiple-item Likert scales.

Due to the diversity of the measures used, and given our aim of presenting the findings in a coherent yet parsimonious framework, we subdivided the domains of therapists' professional and personal qualities into two domains each. The professional domain was divided into (i) taskinstrumental and (ii) socio-emotional domains based on the work by Orlinsky and Howard (1987). The task-instrumental subdomain consists of therapist characteristics and experiences directly and explicitly involved in treatment goals and their pursuit (e.g., usage of therapeutic skills and techniques, as well as difficulties in their employment, and consequent coping strategies). The socioemotional domain includes professional qualities more directly concerned with the therapeutic relationship (e.g., the therapist's relational style or manner and feelings towards patients and about oneself as a therapist).

The personal domain, which pertains mainly to therapists' non-professional or private lives, was similarly divided into (i) intrapersonal and (ii) interpersonal subdomains. The intrapersonal subdomain concerns therapists' experiences in their private lives and of themselves as persons, such as self-identity, attitudes and feelings and internalized concepts such as introjects and object relations. Personality traits and personal qualities or resources such as mindfulness were also categorized as intrapersonal. The interpersonal subdomain, in turn, pertains to characteristics involving relationships to other people. These include relational manner in close relationships, 
interpersonal problems, emotional intelligence, and general social skills. Though these divisions are somewhat arbitrary, they provide a conceptually useful framework for presenting our findings.

\section{Outcome measures.}

Treatment outcome was typically assessed in these studies with symptom-based measures, such as those on depression or on a variety of psychiatric conditions. A few studies used other problem-focused measures, such as those on interpersonal problems, or strength-, resource-, and functioning-oriented measures.

\section{Therapists' Characteristics as Predictors of Outcome}

\section{Professional characteristics.}

\section{Task-instrumental.}

\section{Professional attitudes.}

Sandell and colleagues $(2000,2006,2007)$ studied whether therapist attitudes predicted outcomes within and across predominantly long-term psychodynamic and psychoanalytic treatments (Supplement Table 3). Their findings indicated that valuing insight and kindness as curative factors and viewing therapeutic work as artistry predicted greater patient symptom improvement (Sandell et al., 2006, 2007). Poorer outcomes were predicted by a stronger belief in the curative power of successful adjustment, greater pessimism towards the human condition and the therapeutic enterprise, and a view of therapy more as a set of learnable skills than as artistry (Sandell et al., 2006). Finally, classically psychoanalytic attitudes (e.g., 'neutrality') were less effective in long-term psychodynamic psychotherapy than in psychoanalysis; however, in either treatment, they were not more effective than eclectic attitudes (e.g., valuing both insight and neutrality as well as kindness, supportiveness, and adjustment) (Sandell et al., 2000). A similar result, suggesting congruence or "fit" of professional attitudes and outcomes with the therapy practiced, was found in another smallscale study which saw greater therapist commitment to constructivist epistemology correlate with more client-rated decrease of procrastination in coherence therapy (Toska et al., 2010). 


\section{Subjective efficacy.}

Another core task-instrumental area is how effectively therapists feel able to work toward attaining their goals with patients. This may cover a range of different subjectively experienced abilities, from basic to more advanced relational skills, as well as theoretical and technical knowledge. Using the therapist self-report survey, Development of Psychotherapists Common Core Questionnaire (DPCCQ) (Orlinsky \& Ronnestad, 2005), three studies examined how therapists' selfexperienced skillfulness predicts their outcomes as rated by their patients. The first found that experiencing lower self-reported 'basic relational skills' (e.g., capacity to empathize, naturalness with clients, engaging a working alliance, communicating understanding and concern to patients) was especially harmful in short-term therapy (Heinonen et al., 2012). In these treatments of 12-20 sessions, lesser confidence in these skills predicted no change in therapy. Therapists' lesser confidence in their overall effectiveness and skills predicted poorer outcomes especially in brief treatments at the end of the follow-up period. For therapists who conducted long-term (i.e., 3-year) therapies, such lack of confidence was not similarly detrimental. The second study compared two long-term treatments over 5 years: a 3-year psychodynamic therapy and a 5-year psychoanalysis (Heinonen et al., 2014). In this comparison, therapists' sense of skillfulness predicted faster symptom decrease in long-term psychodynamic therapy compared to psychoanalysis.

The previous studies did not examine interaction effects. However, a Norwegian study of relatively long-term therapies did address these (Nissen-Lie et al., 2013). The impact of therapists' advanced relational skills (defined as therapeutic use of transference and countertransference reactions) was studied while controlling for therapists' interpersonal style, difficulties experienced in practice, and the severity of patients' condition. It turned out that more advanced skillfulness (e.g., use of transference and countertransference reactions) with more severely distressed patients predicted worse outcomes, as measured by patient-rated interpersonal distress. Another study, focusing on the countertransference management of graduate student therapists at a U.S. counseling 
center, found it conversely to predict better therapist- and supervisor-rated outcomes (Gelso et al., 2002). Several of its specific components - such as skills in recognizing boundaries between self and clients, managing own anxiety, and conceptualizing patient and relationship dynamics - also predicted better outcomes.

Looking at the somewhat different construct of therapists' multicultural counseling competencies, assessed by the Multicultural Counseling Inventory (Sodowsky et al., 1994), Larrison and colleagues (2011) found outcome differences between black and white patients predicted by one of the four subscales, the 'relationship' scale - assessing the therapists' openness and comfort working with minorities.

Client feedback may also interact with therapist self-confidence, as shown by a study on the effects of routine patient outcome monitoring applied to various, mostly integrative therapies (de Jong et al., 2012). Therapists with higher confidence in their clinical skills, when also receiving patient feedback, obtained better outcomes than therapists with lower self-efficacy who did not receive feedback. In sum, it appears that therapists' self-rated skillfulness is not a direct indicator of greater effectiveness, but rather that the connections between the two may be complex.

\section{Difficulties in practice and coping mechanisms.}

Two studies investigated whether therapists' difficulties and coping strategies may have a differential impact in different treatment types. In the first, the amount of difficulties experienced in practice did not predict differential outcomes in short-term and long-term therapy (Heinonen et al., 2012). However, therapists' use of avoidant coping (e.g., simply hoping that problems would resolve themselves over time) predicted poorer outcomes in short-term therapy than in long-term therapy. A second study showed that experiencing more frequent difficulties together with more constructive coping (e.g., discussing the problem with the patient or a colleague, seeking supervision, selfreflection) predicted faster symptom reduction in long-term psychotherapy as compared to psychoanalysis (Heinonen et al., 2014). 
Several Norwegian studies have also studied therapists' experiences of difficulties in practice. One found that a specific type of difficulty which Orlinsky and Rønnestad (2005) termed 'negative personal reaction' (e.g., difficulties in liking and respecting a patient, and tolerating a patient's emotional needs) predicted worse patient outcomes, as measured by patient self-rated interpersonal distress (Nissen-Lie et al., 2013). Likewise, better outcomes were predicted by therapists' use of constructive coping strategies, such as self-reflection, consultation with colleagues, or problemsolving with a patient. In this study, poorer outcomes were also predicted by the therapist's use of 'avoidant coping' strategies. More surprisingly, however, better outcomes were predicted by another type of difficulties that Orlinsky and Rønnestad (2005) described as 'professional self-doubt' (e.g., feeling unsure how best to help a patient).

In an effort to replicate these findings of Nissen-Lie and colleagues on a less experienced sample (i.e., therapist trainees conducting cognitive-behavioral therapy), Odyniec and colleagues (2017) found an opposite pattern. That is, beginning therapists' tendency to experience 'negative personal reaction' towards patients surprisingly predicted better outcomes. In turn, professional selfdoubt predicted worse outcomes. In line with the latter result and also focusing on trainees, Swift and colleagues (2018) investigated anticipated outcomes by clients and therapists prior to treatment for 34 clients. Both client and therapist expectations predicted eventual treatment effectiveness, although only the therapists' expectations that their specific clients - as opposed to their clients in general would make positive gains was predictive of better outcomes.

\section{Socio-emotional characteristics.}

\section{Interpersonal abilities.}

Two measures of therapists' interpersonal abilities have been developed recently (Anderson et al., 2009; Schöttke et al., 2017). Although their scope overlaps with the previously reviewed domain of task-instrumental skillfulness, both comprise qualities that are central to helping relationships across 
numerous occupations. Methodologically, they also differ from the previously reviewed instruments of therapist characteristics by being performance-based and rated by external observers.

Anderson and colleagues' Facilitative Interpersonal Skills (FIS) (2009) assesses therapists' verbal fluency and responsiveness, emotional expressiveness, persuasiveness, warmth, positive regard, hopefulness, empathy, and capacity to form and repair alliances. These qualities are rated by observers in response to video-taped, simulated, challenging patient cases. The composite score of these skills predicted better OQ-45 outcomes of 25 experienced therapists seeing 1,141 clients for a mean of 9 sessions, in the first study by Anderson and colleagues (2009). In a subsequent study (Anderson, McClintock, Himawan, Song, \& Patterson, 2016), greater symptom reduction was achieved by therapist trainees who were assessed to be higher in FIS over a year prior to starting clinical work. However, the effect depended on the treatment duration, being observed in therapies of 4,8 and 12 sessions but becoming attenuated in therapies of 16 sessions and no longer evident in those of 20 sessions. Recently using an innovative design, Anderson, Crowley et al. (2016) also randomized patients for up to 7 sessions with both therapist and non-therapist (e.g., biology, chemistry, history) doctoral students who were higher and lower in FIS. Students higher on the FIS measure achieved better outcomes on symptom and global measures at post-treatment and/or 3months' follow-up, regardless of their prior training.

Using a similar methodology, Schöttke and colleagues' (2017) assessed therapist qualities in a performance task, consisting of a group discussion after viewing a therapeutic intervention video clip intended to provoke debate. The observer-rated group measure TRIB-G (Therapy-Related Interpersonal Behaviors-Group Format) assessed largely similar qualities as FIS: Clear and positive communication, empathy and affective attunement, respect and warmth, managing criticism, and willingness to cooperate. Higher TRIB-G scores among therapist trainees predicted better outcomes in both cognitive-behavioral and psychodynamic therapies.

\section{Relational manner and feelings.}


Two studies based on self-report questionnaires have investigated whether therapists' typical relational manner and feelings during therapy sessions may have differential impacts in different durations and types of therapies. In the first, no differences in outcome were observed between shortand long-term therapies in relation to therapists' 'affirming' (i.e., accepting, warm, friendly, tolerant) professional relational manner (Heinonen et al., 2012). In contrast, a second study comparing outcomes in long-term psychodynamic therapy and psychoanalysis found that therapists conducting psychoanalysis who reported having a less 'affirming' manner with patients obtained better outcomes (Heinonen et al., 2014). This finding fits with the earlier findings of Sandell (2000), who observed that a classically neutral stance was harmful in long-term psychodynamic therapy, but not in psychoanalysis.

Both studies also explored how therapists' typical feelings during therapy sessions (assessed by multiple item scales of 'flow', anxiety, and boredom) predict therapy outcomes. In the first one, therapists experiencing less flow during sessions (feeling stimulated, engrossed, inspired, and challenged) obtained poorer outcomes in short- than in long-term therapy (Heinonen et al., 2012). In the second study, more frequently experienced 'flow' but also more frequently experienced 'boredom' predicted faster symptom reduction in long-term psychodynamic therapy as compared to psychoanalysis (Heinonen et al., 2014). Flow and boredom appear incompatible with one another and the results therefore contradictory. However, it should be noted that therapists were reporting on their typical feelings, rather than feelings with the particular patients whose outcome was studied.

In another study (Schöttke et al., 2017), the work motivation or commitment of therapist trainees (consisting of enthusiasm and interest in one's therapy approach, patients, and personal selfreflection) was assessed by interview. However, this variable failed to predict outcomes in cognitivebehavioral or psychodynamic treatments.

\section{Personal characteristics.}


The characteristics of therapists in their non-professional or private lives have been subdivided into intrapersonal (describing how a person relates to him- or herself) and interpersonal (describing a person's way of communicating with and relating to others) qualities. Note that this distinction is not absolute and the two aspects may be interlinked.

\section{Intrapersonal qualities.}

\section{Internalized self-other representations.}

Seven studies have investigated the relation of therapy outcome to therapists' intrapersonal internalized images of self and others, such as (i) introjects or stable ways of self-response (Bruck et al., 2006; Nissen-Lie et al., 2017), (ii) attachment patterns (Bruck et al., 2006; Cologon, Schweitzer, King, \& Nolte, 2017; Lawson \& Brossart, 2003; Muratori et al., 2017; Schauenburg et al., 2010), and (iii) perceptions of early parental care (Hilliard et al., 2000). Bruck et al. (2006), using the SASB Intrex questionnaire, studied whether therapists' introjects and their attachment styles predict therapy process and outcome. A more 'affiliative' introject (i.e., more self-loving attitude) in therapists did predict a greater decrease in patient-rated target complaints. Likewise, a secure attachment style predicted greater decrease in therapist-rated interpersonal distress and therapist-rated target complaints. Therapists' insecure attachment, on the other hand, predicted generally worse patient outcomes: Therapists' 'fearful attachment' was associated with greater therapist-rated interpersonal problems and poorer patient-rated global functioning. A 'preoccupied attachment' pattern also predicted poorer patient-rated global functioning (but, unexpectedly, greater symptom decrease). Finally, a 'dismissive attachment' was associated with increased levels of both therapist- and patientrated interpersonal problems. It should be noted that shared method variance may have inflated correlations when therapists assessed both their own characteristics and treatment outcomes.

Another study (Nissen-Lie et al., 2017) that used the SASB Intrex examined how the interaction between therapists' loving attitude toward themselves as persons and the type of treatment difficulty described as 'professional self-doubt' relates to outcome. The greatest decrease 
in patient-rated interpersonal problems was predicted by a combination of "loving oneself as a person and doubting oneself as a therapist". In contrast to Bruck and colleagues' findings, therapists' Intrex-assessed self-love did not directly predict outcome. Schauenburg et al. (2010) also did not find therapists' 'secure attachment' by itself related to outcomes in inpatient treatment cases. However, a statistically significant interaction effect was noted, indicating therapists' secure attachment to predict better outcomes for patients with greater pre-treatment interpersonal distress. In complement to this finding, therapists' preoccupation with relationships was shown to predict less reduction of aggression in children treated for behavioral problems (Muratori et al., 2017).

Closely related to these studies on attachment, Hilliard et al. (2000) studied how therapists' perceptions of their early relationships with parents (presumably functioning as a template for their later relationships) predicted patient outcome in short-term psychodynamic therapy. A warmer relationship with parents was not directly associated with outcome as assessed by either patients, outside observers, or therapists. Nevertheless, as early parental relations predicted therapists' own ratings of the therapy process, which in turn predicted their outcome ratings, the authors hypothesized an indirect link between therapists' early care-giving relationships and outcome. Also Hersoug (2004) failed to find a direct association between therapists' perceptions of early parental care and change in patients' maladaptive defenses.

\section{Personality traits and psychological vulnerabilities and resources.}

Other studies have investigated various potential psychological vulnerabilities and resources, such as neuroticism (Rieck \& Callahan, 2013), emotional intelligence (Kaplowitz et al., 2011; Rieck \& Callahan, 2013), mindfulness and resilience (Pereira et al., 2017; Ryan et al., 2012; Stanley et al., 2006), as well as reflective functioning (Cologon et al., 2017). The current review found only one study published after 2000 on relating treatment outcome to therapist characteristics on the traditional Big Five personality dimensions (Rieck \& Callahan, 2013). Of the five global selfreported personality dimensions assessed by the NEO-FFI, only 'neuroticism' predicted trainee 
therapists' good outcomes on the OQ-45 in therapies ranging from 29 to 39 sessions. Neuroticism further interacted with 'emotional intelligence' measured by a performance-based task, such that neuroticism contributed more positively to outcome if therapists were higher in emotional intelligence. It may be that for more 'emotionally intelligent' trainees, neuroticism (or sensitivity to negative emotional states) is an aid to understanding and empathizing with their patients.

Therapists' 'emotional intelligence' (EI) was also investigated in a pilot study of 23 therapists and patients conducting cognitive-behavioral and brief relational therapy (Kaplowitz et al., 2011). In this small sample, borderline but statistically non-significant positive associations $(\mathrm{p}<.10)$ were reported between emotional intelligence, its subcomponents, and outcome: showing total EI and its subcomponent, capacity to integrate emotion, to predict decrease in therapist-rated target complains and interpersonal complaints, and the capacity to manage emotion to predict decrease in patient-rated interpersonal problems. A third EI component, understanding of emotion, significantly predicted a reduction in therapist-rated target complaints and interpersonal problems.

A concept linked with emotional intelligence is mindfulness, understood as the capacity to bring one's attention to the present moment with complete acceptance and without judgment (Ryan et al., 2012). For example, Ryan et al. (2012), using the Kentucky Inventory of Mindfulness Skills, found higher levels of therapist mindfulness to predict a greater decrease in patient-rated interpersonal problems, but not global symptomatology, in cognitive-behavioral and brief relational therapies lasting 30 sessions. This differed from earlier findings by Stanley et al. (2006), who paradoxically found greater therapist mindfulness (assessed by the Mindfulness Awareness Attention Scale) associated with poorer global functioning in mostly cognitive-behavioral analysis system psychotherapy (CBASP). Pereira et al. (2017), on the other hand, found that better outcomes were associated with the practitioners' higher degree of mindfulness and resilience as well as their combination. In another study (Larrison et al., 2011), therapist burnout was not predictive of 
outcome differences between black and white clients; neither was these therapists' non-prejudice, i.e., a tendency to focus on similarities rather than differences between the self and others.

'Reflective functioning' (i.e., the capacity to conceptualize, identify, and understand one's own and others' mental states) is a concept that overlaps with mindfulness and emotional intelligence. Cologon and colleagues (2017) found that higher levels of reflective functioning in 25 therapists treating 1,001 patients for a mean of 5 (range 2-43) sessions predicted better outcomes in varied therapeutic approaches. They also noted an interaction effect between therapist variables, similar to the findings reported earlier by Rieck and Callahan (2013) and Nissen-Lie et al. (2017). The authors found reflective functioning especially beneficial for the outcomes of therapists who were higher in 'insecure attachment' but that high attachment insecurity was associated with worse outcomes for therapists who had lower levels of reflective functioning.

\section{Interpersonal qualities.}

Relational manner in personal life.

Dinger et al. (2007) conducted a study of German inpatients receiving individual therapy as part of a multimodal treatment program, in which therapists' interpersonal dispositions in their personal relationships to behave 'too dominantly' or 'too friendly' (e.g., in a self-sacrificing, overly accommodating, or intrusive manner) were unrelated to improvements in patients' general psychiatric symptoms. Nevertheless, in a moderator analysis, the positive effect of having a good alliance on outcome was stronger for therapists who described themselves as too 'cold' in private life as compared to those who viewed themselves as 'too friendly'.

The type and length of psychotherapy may be another moderating factor, as suggested by two other studies. In the first, therapists who viewed themselves as interpersonally engaged and outgoing in their personal lives produced faster symptom reduction in short-term than in long-term therapies (Heinonen et al., 2012). In contrast, therapists who viewed themselves as less intrusive and more considerate produced greater symptom reduction in long-term than in short-term psychotherapy. In 
the second study (Heinonen et al., 2014), therapists who viewed themselves as less 'forceful' (e.g., directive, demanding, intense) and more 'genial' (e.g., warm, receptive, tolerant) in private life produced faster symptom reduction in long-term therapy as compared to psychoanalysis. Paradoxically in psychoanalysis therapists whose manner was characterized by being more forceful and less 'aloof' (e.g., less cold, guarded, and reserved) seemed to produce more favorable outcomes.

Finally, in a study extending prior research on therapists' early relationship to their parents, Lawson and Brossart (2003) studied how therapist trainees' current relationships with their parents predicted treatment outcome. Investigating various therapeutic approaches in a year-long practicum, no associations were found between treatment outcome and how autonomous or enmeshed trainees were with their parents.

\section{Relational capacities in personal life.}

Bambling and King (2013) assessed 92 therapists on their social skills, conceptualized as capacities for emotional and social expressivity, sensitivity, and regulation, as measured by the Social Skills Inventory (SSI). In an 8-session, extended problem-solving treatment (a cognitive-behaviorally oriented brief intervention) greater social skills predicted marginally significantly $(p=.06)$ decreases in patient-rated depressive symptoms.

In studies of trainee therapists at a training clinic in the U.S. (Anderson, McClintock et al., 2016), and of experienced, mainly doctoral-level therapists at a university counseling center (Anderson et al., 2009), social skills measured by the SSI failed to predict patient-rated outcomes. A measure of sociability (i.e., how outgoing and involved one is in relationships) was not associated with outcome either (Anderson, McClintock et al., 2016). However, a third investigation studying both therapist and non-therapist doctoral students found self-rated social skills and a measure for empathy (Gough, 1987) to predict decreases in OQ-45 symptoms (Anderson, Crowley et al., 2016).

While the previous studies all relied on therapist-rated measures to assess therapist characteristics, Schöttke et al. (2017) used an interview to evaluate therapist trainees' interpersonal 
capacities such as quality of self- and other-perception and communication skills, but no relationship of these variables to outcomes was found. Overall, therapists' social skills in their private life therefore have yielded relatively weak or inconsistent associations with patient outcomes.

\section{Discussion}

Psychotherapists are known to differ in their effectiveness (Johns et al., 2019). The present challenge is to provide content to this observation: What are the characteristics of the psychotherapist that actually contribute to these differences? In pursuing this question, we reviewed studies published since 2000 to establish which dispositional or stable psychotherapist characteristics (i.e., conceived of as existing regardless of the individual client seen) predict better or worse treatment outcomes.

The review covered 31 studies meeting our selection criteria and investigating psychotherapy in the treatment of various mental health problems in all age groups. The studies varied notably in the types of treatment examined (i.e., therapeutic approaches and methods), duration of therapy, assessment measures, length of follow-up, and statistical techniques. This variety, together with the still relatively small number of studies in the field, cautions against firm conclusions or clinical recommendations. Nonetheless, it is worth summarizing our findings, and highlighting the emerging, intersecting, and promising themes for further research, as well as the potential implications for the selection of psychotherapy trainees, training, supervision, and clinical practice. In discussing the findings, we continue to use the division between professional and personal characteristics, and a further subdivision into task-oriented and socio-emotional and intra- and interpersonal qualities (Table 1). Not all the study findings were considered equally reliable. We have accordingly put more emphasis on the studies with the highest quality: that is, where outcomes were rated by clients; with reliable and valid measurement of therapist characteristics; and predicting outcomes using multilevel modeling techniques that take the nested structure of patient-therapist data into account. In brief, the attributes of therapists with the strongest relationship to client outcome were the professional ones, i.e., more directly pertaining to the work of conducting psychotherapy. The more global or personal 
variables relating to life outside of therapy typically did not have a strong direct impact on outcome; rather, they interacted with other moderators pertaining to the work of therapy and the therapeutic relationship.

Among professional, task-oriented characteristics, therapists' work-related values, attitudes, and treatment philosophy function as the foundation for the interventions that therapists use and the kinds of relationship they seek to create with their clients. However, interest in professional values in outcome research has waned since the 1970s and 1980s (cf. Beutler et al., 2004). The little extant research suggests that in long-term psychodynamic therapies valuing insight and kindness as curative factors may lead to better outcomes (Sandell et al., 2006, 2007).

Difficulties may arise during therapy and therapists cope with these in varying ways. However, therapists' own evaluations of their skills, difficulties, and coping strategies do not appear to be obvious or consistent predictors of outcome. Despite this, experienced therapists' confidence in their skills may facilitate change, especially in short-term psychotherapies (Heinonen et al., 2012). However, in longer-term therapies, certain types of self-reported skills - such as confidence in using one's own emotional reactions to patients - have predicted worse outcomes, especially when treating more disturbed clients (Nissen-Lie et al., 2013). In these studies, therapists' 'professional self-doubt' has been found to be beneficial (Nissen-Lie et al., 2013, 2017). The positive effect of professional self-doubt resonates with findings of earlier research on therapists, for example Najavits and Strupp (1994), who found that effective therapists reported having made more mistakes than less effective ones. Yet other studies and measures of self-doubt show no association to client outcomes (Sandell et al., 2006, 2007) and studies with relatively inexperienced therapists have shown self-doubt related to poorer outcomes (Odyniec et al., 2017, see also Swift et al., 2018). One reason for these inconsistencies may be that self-ratings are likely influenced by unmeasured biases due to social desirability, personality, or even cultural factors: these effects, in turn, may depend on other variables, such as level of clinical experience and therapy method used. Therefore, looking at 
moderators of the relationship between professional self-concept(s) and outcome may clarify their inconsistent associations.

Therapists' relational qualities in their professional role were classified under the rubric of 'socio-emotional characteristics'. These rater-assessed socio-emotional abilities appeared to be more consistent outcome predictors than therapist self-ratings of therapeutic skills. An aggregate of qualities such as empathy, warmth and positive regard, clear and positive communication, and capacity to manage criticism have predicted better outcomes of experienced therapists (Anderson et al., 2009), trainees (Anderson, McClintock et al., 2016; Schöttke et al., 2017), and both therapist and non-therapist doctoral students (Anderson, Crowley et al., 2016). The last two findings would suggest that these may be some of the "natural qualities" or "talent" that effective therapists bring to their profession from the start. Whether they can also be developed through training, or if they should be used as part of a set of empirically validated selection criteria for entry into therapist training, seems a central question for future longitudinal studies (Orlinsky et al., 2015).

The reviewed studies also suggest that an interpersonally challenging situation may be what is needed to bring out therapist facilitative qualities. Only in such situations are capacities such as self-control, emotional containment, and empathy perhaps properly tested, and the various resources differentiating the more from the less effective therapists emerge. This suggestion is also indirectly supported by (unreviewed) qualitative research on high-performing therapists (Moltu, Binder, \& Nielsen, 2010) and work on therapists' countertransference management (Hayes et al., 2018).

The studies in this review suggest that various measures of self-relatedness (such as quality of introjects, attachment style, or experience of parental bonding) have little direct effect on patient outcome. These concepts by themselves may be too distant from the treatment setting to have a detectable impact on patient change (Wolff \& Hayes, 2009). In other words, they may not become activated during treatment, diminishing their predictive powers. Even if activated, their effect would 
expectably depend on therapists' capacity for differentiating their selves from the patient and on their self-control, self-insight and countertransference management (Hayes et al., 2018).

Indeed, several findings point toward this view, such as the finding that therapists' professional self-doubt (interpreted as modesty or 'humility') interacted with a nurturing attitude towards themselves to produce the best outcomes (Nissen-Lie et al., 2017). The finding that securely attached therapists were more effective with more disturbed patients is another example (Schauenburg et al., 2010). When faced by distressing or provocative patient behaviors, therapists' positive resources may help them remain flexible and focused on the client (cf. Lopez \& Brennan, 2000; Mallinckrodt, 2000). In a similar vein, some studies provide support for the benefits of therapists' personal qualities as mindfulness (Ryan et al., 2012), emotional intelligence (Rieck \& Callahan, 2013), and reflective functioning (Cologon et al., 2017). Several studies likewise suggest that therapists' psychological resources help compensate for their psychological vulnerabilities, for example when therapists with higher 'neuroticism' as well as emotional intelligence (EI) were more effective than those higher in neuroticism but lower in EI (Rieck \& Callahan, 2013). It is plausible that those therapists were able to use their heightened sensitivity towards negative affect, embedded in trait neuroticism as a 'mentalizing' or empathizing capacity in service of the patient. Similarly, high reflective functioning in therapists was found to compensate for their insecure attachment, and secure attachment to compensate for lower reflective functioning in predicting more favorable outcomes (Cologon et al., 2017).

Overall, therapists' self-rated interpersonal qualities in their private life (interpersonal problems, social skills, and relational manner) showed little independent association with psychotherapy outcomes. What may explain the lack of findings in this realm?

One possibility is that therapists adjust their professional demeanor from their manner in personal relationships to match their preferred treatment model, attenuating the predictive power of the personal element (Heinonen \& Orlinsky, 2013). It may also be that people who choose to become 
therapists differ from the general population in their interpersonal characteristics; thus, standard population measures may not capture the relevant differences between therapists. As most of the studies of personal characteristics were based on therapists' self-reports, one could also wonder whether assessments made by partners or friends of therapists would be better predictors of outcome.

Notwithstanding the difficulties in disentangling the professional versus private aspects of therapists' relational qualities (and their interplay), focusing on specific therapists' interpersonal capacities seems a promising avenue for future research. A similar conclusion was reached by Lingiardi et al. (2018) in their recent review, focusing on therapist characteristics solely in psychodynamic therapies, and sharing 12 studies in common with the present review. Both reviews have also observed many other characteristics (e.g., attachment patterns) to not show direct effects but only impact outcome in interaction with other factors, such as client factors (e.g., symptomatic impairment), or measures of the therapeutic process (e.g., therapeutic alliance). Hence, both reviews point to the complexity of how therapists interact as professionals and people overall with the numerous variables linked to outcome, and contraindicate the study of therapists in a 'vacuum'.

\section{Limitations}

Some limitations of the individual studies have already been noted. In addition, there are four general practical and conceptual concerns with the studies: (1) the number of studies and the file drawer problem, (2) sample size issues, (3) statistical analyses, and (4) the dyadic nature of psychotherapy. First, there were too few studies in each subdomain of therapist characteristics to conduct a metaanalysis of the strength of their predictive value on outcome. It follows that moderator analyses of possibly important factors, such as therapy type or duration, were even less feasible. Also, it is hard to evaluate the extent of the 'file-drawer problem'. Do the published studies represent those few where significant associations were found? Second, the sample sizes in most studies that met our inclusion criteria were not great, with the risk of not having enough therapists (or patients per therapist) for adequate power to detect associations between therapist characteristics and outcome. If 
the number of patients per therapists is too low, the risk is that patient variability explains most of the differences found between therapist characteristics and outcome. Several suggestions exist on the appropriate sample sizes for multilevel modeling of therapist and patient effects on outcome (Adelson \& Owen, 2012). It is safe to say that the higher the number of therapists as well as patients per therapist, the less the risk of biased or spurious effects.

Third, only 13 of the 31 studies applied multilevel modeling to disaggregate outcome variance into therapist and patient variability. This might result in underestimating the standard errors of therapist factors, an inflated Type I error rate, and spuriously identified therapist predictors (Raudenbush \& Bryk, 2002). Fourth, the dyadic nature of psychotherapy underlines some common practical and conceptual concerns in this area. Most concretely, many studies still heavily rely on a single viewpoint, typically the therapist's, in assessing clinician qualities. To uncover the determinants of therapist effects, future studies would benefit from triangulating the perspectives of self-report, patient evaluations, and performance-based measurement. Conceptually related to this, mapping therapists' dispositional characteristics to patterns of patient change undoubtedly runs into the danger of reducing the therapist to a 'variable' without considering the interplay in which the disposition come into play. It may therefore lose sight of the therapeutic dyad as an ongoing coconstruction of connection and meaning which exists to facilitate growth for the client, but also influences the therapist in a reciprocal manner (Orlinsky \& Howard, 1987).

Relatedly also, the concept of appropriate responsiveness (Kramer \& Stiles, 2015; Stiles, 2009) can be useful for understanding the lack of relationship between many examined therapist variables and client change. Responsiveness can be defined as the "continual adjustment of responses based on the evolving nature of the interpersonal situation", where each member of the dyad adjusts his or her responses to the other, guided by his or her particular goals for the interaction (Hatcher, 2015; Stiles, 2009). For the therapist, responsiveness may be thought of as a "metacompetency (...) tying together a number of lower order natural skills and competencies such as executive 
functioning, reflection, and interpersonal competencies" (Hatcher, 2015). Insofar as therapy outcomes hinge on micro-attuned responsiveness, attempts to map therapists' stable characteristics to client outcomes may be futile, unless the disposition relates to the overarching meta-competency of appropriate responsiveness.

Last but not least, an implicit assumption in much of the reviewed literature - and this review - is that therapist effects are largely due to variability in traits rather than states. However, staterelated fluctuations may also occur in therapists' tendencies, dispositions, or their expression, as well as clinician effectiveness. For instance, troubling circumstances in one's private (e.g., illness in family) or professional life (e.g., particularly difficult clients, poor workplace support) may overburden the therapist's intra- and interpersonal capacities for effectively relating to clients, leading to lower effectiveness than under more favorable or typical circumstances; similarly, a particularly helpful consultation from a colleague may have the opposite effect.

\section{Conclusions}

According to the consistent findings of this review, more effective therapists are characterized by interpersonal capacities that are professionally cultivated but likely rooted in their personal lives and attachment history- such as empathy, verbal and non-verbal communication skills and capacity to form and repair alliances - especially with interpersonally challenging clients (see also, Johns et al., 2019). Several studies suggest that therapists' self-rated skillfulness, difficulties in practice, coping mechanisms, and attitudes towards therapy may matter too, but not always in a linear or expected direction. No clear evidence exists for the consistent superiority of any particular (Big Five) personality style. However, several recent studies point towards the importance of the therapist's basic relational skills and a warm interpersonal style. As these skills can already be observed in trainees and even non-therapists, they may represent the 'natural talent' that clinicians bring in varying measure to their professional work (e.g., Orlinsky \& Ronnestad, 2005; Nissen-Lie \& Orlinsky, 2014)). Having a personal 'secure attachment' style, a capacity to tolerate intense displays 
of dysphoric affect, hostility and aggression, and an ability to stay focused on the client, also seem important, particularly in treating more disturbed clients.

The apparent paradoxes emerging in some of the studies, such as a beneficial effect of the difficulty in practice termed 'professional self-doubt', and a potentially detrimental effect of 'advanced interpersonal skills' (transference and countertransference work) with more disturbed patients, points to the direction of modesty or humility as a potential virtue in therapeutic work. This corresponds with the concept of 'cultural humility' in psychotherapists as a prerequisite for successful practice with cultural and ethnic minorities (Owen et al., 2016). Still, it is important to note that some studies have not found such associations, and one is left with hypotheses rather than conclusions. This lack of consistency suggests that the effects of therapist dispositions likely are sensitive to the context in which they are studied, including the type of treatment, participants, and the measures that are involved.

This review has identified some promising lines of research. It also underlines the need for future research to study therapist outcome differences across different treatment models and modalities with appropriate designs and methods. It is essential that future studies of therapist factors use sufficient number of therapists, patients per therapist, longitudinal measurement, and triangulate different observational perspectives on therapist dispositions and capacities (including self-report, patient report, peer or supervisor report, and performance-based evaluations). We believe that sophistication as well as breadth in the analyses including appropriately disaggregating variability, quantitative micro-process studies, and in-depth qualitative work is necessary to bring the field forward. It is also important to understand how these more or less stable attributes of therapists are manifested dynamically through interaction with individual patients' pathology, personality, interpersonal style, and other qualities that determine the course of treatment. 


\section{References}

(References marked with an asterisk indicate studies included in the review.)

Adelson, J. L., \& Owen, J. (2012). Bringing the psychotherapist back: Basic concepts for reading articles examining therapist effects using multilevel modeling. Psychotherapy, $49,152-162$.

*Anderson, T., Crowley, M. E. J., Himawan, L., Holmberg, J. K., \& Uhlin, B. D. (2016). Therapist facilitative interpersonal skills and training status: A randomized clinical trial on alliance and outcome. Psychotherapy Research, 26, 511-529.

*Anderson, T., McClintock, A. S., Himawan, L., Song, X., \& Patterson, C. L. (2016). A prospective study of therapist facilitative interpersonal skills as a predictor of treatment outcome. Journal of Consulting and Clinical Psychology, 84, 57-66.

*Anderson, T., Ogles, B. M., Patterson, C. L., Lambert, M. J., \& Vermeersch, D. A. (2009). Therapist effects: Facilitative interpersonal skills as a predictor of therapist success. Journal of Clinical Psychology, 65, 755-768.

Anderson, T., \& Patterson, C. (2013). Facilitative interpersonal skill task and rating method. Unpublished manuscript. Retrieved June 26, 2018 from http://clinica.ispa.pt/ficheiros/areas_utilizador/user11/facilitative_interpersonal_skill_ma $\underline{\text { nual_and_rating_scale.pdf }}$ 
Baldwin, S. A., \& Imel, Z. E. (2013). Therapist effects: Findings and methods. In M. J. Lambert (Ed.), Bergin and Garfield's handbook of psychotherapy and behavior change. (6th ed.) (pp. 258-39). Hoboken, NJ: Wiley.

*Bambling, M., \& King, R. (2013). Extended problem solving treatment for depression. Counselling \& Psychotherapy Research, 13, 317-323.

Barkham, M., Lutz, W., Lambert, M. J., \& Saxon, D. (2017). Therapist effects, effective therapists, and the law of variability. In L. G. Castonguay, \& C. E. Hill (Eds.), How and why are some therapists better than others: Understanding therapist effects (pp. 13-23). Washington, DC: American Psychological Association.

Beutler, L. E., Machado, P. P. P., \& Neufeldt, S. A. (1994). Therapist variables. In A. E. Bergin, \& S. L. Garfield (Eds.), Handbook of psychotherapy and behavior change. (4th ed.) (pp. 229-269). Oxford: Wiley.

Beutler, L. E., Malik, M., Alimohamed, S., Harwood, T. M., Talebi, H., Noble, S., \& Wong, E. (2004). Therapist variables. In M. J. Lambert (Ed.), Bergin and Garfield's handbook of psychotherapy and behavior change. (5th ed.) (pp. 227-306). New York: Wiley.

*Bruck, E., Winston, A., Aderholt, S., \& Muran, J. C. (2006). Predictive validity of patient and therapist attachment and introject styles. American Journal of Psychotherapy, 60, 393-406.

Castonguay, L. G., \& Hill, C. E. (Eds.) (2017). How and why are some therapists better than others? Understanding therapist effects. Washington, DC: American Psychological Association. 
*Cologon, J., Schweitzer, R. D., King, R., \& Nolte, T. (2017). Therapist reflective functioning, therapist attachment style and therapist effectiveness. Administration and Policy in Mental Health and Mental Health Services Research, 44, 614-625.

Crits-Christoph, P., Baranackie, K., Kurcias, J. S., \& Beck, A. T. (1991). Meta-analysis of therapist effects in psychotherapy outcome studies. Psychotherapy Research, 1, 81-91.

*de Jong, K., van Sluis, P., Nugter, M., Heiser, W., \& Spinhoven, P. (2012). Understanding the differential impact of outcome monitoring: Therapist variables that moderate feedback effects in a randomized clinical trial. Psychotherapy Research, 22, 464-474.

*Dinger, U., Strack, M., Leichsenring, F., \& Schauenburg, H. (2007). Influences of patients' and therapists' interpersonal problems and therapeutic alliance on outcome in psychotherapy. Psychotherapy Research, 17, 148-159.

Gelso, C. J., \& Hayes, J. A. (2007). Countertransference and the therapist's inner experience: Perils and possibilities. Mahwah, NJ: Lawrence Erlbaum.

*Gelso, C. J., Latts, M. G., Gomez, M. J., \& Fassinger, R. E. (2002). Countertransference management and therapy outcome: An initial evaluation. Journal of Clinical Psychology, 58, 861-867.

Goldberg, S. B., Babins-Wagner, R., Rousmaniere, T., Berzins, S., Hoyt, W. T., Whipple, J., . . Wampold, B. E. (2016). Creating a climate for therapist improvement: A case study of an agency focused on outcomes and deliberate practice. Psychotherapy, 53, 367-375.

Goldberg, S. B., Rousmaniere, T., Miller, S. D., Whipple, J., Nielsen, S. L., Hoyt, W. T., \& Wampold, B. E. (2016). Do psychotherapists improve with time and experience? A 
longitudinal analysis of outcomes in a clinical setting. Journal of Counseling Psychology, 63, 1-11.

Hatcher, R. L. (2015). Interpersonal competencies: Responsiveness, technique, and training in psychotherapy. American Psychologist, 70, 747-757.

Hayes, J. A., Gelso, C.J, Goldberg, S. B, Kivlighan, M. (2018). Countertransference management and effective psychotherapy: Meta-analytic findings. Psychotherapy Theory Research \& Practice, 55, 496-507.

*Heinonen, E., Knekt, P., Jääskeläinen, T., \& Lindfors, O. (2014). Therapists' professional and personal characteristics as predictors of outcome in long-term psychodynamic psychotherapy and psychoanalysis. European Psychiatry, 29, 265-274.

*Heinonen, E., Lindfors, O., Laaksonen, M. A., \& Knekt, P. (2012). Therapists' professional and personal characteristics as predictors of outcome in short- and long-term psychotherapy. Journal of Affective Disorders, 138, 301-312.

Heinonen, E., \& Orlinsky, D. E. (2013). Psychotherapists' personal identities, theoretical orientations, and professional relationships: Elective affinity and role adjustment as modes of congruence. Psychotherapy Research, 23, 718-731.

*Hersoug, A. G. (2004). Assessment of therapists' and patients' personality: Relationship to therapeutic technique and outcome in brief dynamic psychotherapy. Journal of Personality Assessment, 83, 191-200. 
*Hilliard, R. B., Henry, W. P., \& Strupp, H. H. (2000). An interpersonal model of psychotherapy: Linking patient and therapist developmental history, therapeutic process, and types of outcome. Journal of Consulting and Clinical Psychology, 68, 125-133.

*Kaplowitz, M. J., Safran, J. D., \& Muran, C. J. (2011). Impact of therapist emotional intelligence on psychotherapy. Journal of Nervous and Mental Disease, 199, 74-84.

Kramer, U., \& Stiles, W. B. (2015). The responsiveness problem in psychotherapy: A review of proposed solutions. Clinical Psychology: Science and Practice, 22, 277-295.

Kraus, D. R., Bentley, J. H., Alexander, P. C., Boswell, J. F., Constantino, M. J., Baxter, E. E., \& Castonguay, L. G. (2016). Predicting therapist effectiveness from their own practice-based evidence. Journal of Consulting and Clinical Psychology, 84, 473-483.

Johns, R. G., Barkham, M., Kellett, S., Saxon, D. (2019). A systematic review of therapist effects: A critical narrative update and refinement to Baldwin and Imel's (2013) review, Clinical Psychology Review, 67, 78-93.

Lambert, M. J. (2013). The efficacy and effectiveness of psychotherapy. In M. J. Lambert (Ed.), Bergin and Garfield's handbook of psychotherapy and behavior change (6th ed.) (pp. 169-219) Hoboken, NJ: Wiley.

*Larrison, C. R., Schoppelrey, S. L., Hack-Ritzo, S., \& Korr, W. S. (2011). Clinician factors related to outcome differences between black and white patients at CMHCs. Psychiatric Services, 62, 525-531.

*Lawson, D. M., \& Brossart, D. F. (2003). Link among therapist and parent relationship, working alliance, and therapy outcome. Psychotherapy Research, 13, 383-394. 
Lingiardi, V., Muzi, L., Tanzilli, A., \& Carone, N. (2018). Do therapists' subjective variables impact on psychodynamic psychotherapy outcomes? A systematic literature review. Clinical Psychology \& Psychotherapy, 25, 85-101.

Lopez, F. G., \& Brennan, K. A. (2000). Dynamic processes underlying adult attachment organization: Toward an attachment theoretical perspective on the healthy and effective self. Journal of Counseling Psychology, 47, 283-300.

Mallinckrodt, B. (2000). Attachment, social competencies, social support, and interpersonal process in psychotherapy. Psychotherapy Research, 10, 239-266.

Moltu, C., Binder, P., \& Nielsen, G. H. (2010). Commitment under pressure: Experienced therapists' inner work during difficult therapeutic impasses. Psychotherapy Research, 20, 309-320.

*Muratori, P., Polidori, L., Chiodo, S., Dovigo, V., Mascarucci, M., Milone, A., ... \& Lochman, J. E. (2017). A pilot study implementing Coping Power in Italian community hospitals: Effect of therapist attachment style on outcomes in children. Journal of Child and Family Studies, 26, 3093-3101.

Najavits, L., \& Strupp, H. H. (1994). Differences in the effectiveness of psychodynamic therapies: A process-outcome study. Psychotherapy, 31, 114-123.

Nissen-Lie, H. A., Goldberg, S. B., Hoyt, W. T., Falkenstrom, F., Holmqvist, R., Nielsen, S. L., \& Wampold, B. E. (2016). Are therapists uniformly effective across patient outcome domains? A study on therapist effectiveness in two different treatment contexts. Journal of Counseling Psychology, 63, 367-378. 
*Nissen-Lie, H. A., Monsen, J. T., Ulleberg, P., \& Ronnestad, M. H. (2013). Psychotherapists' self-reports of their interpersonal functioning and difficulties in practice as predictors of patient outcome. Psychotherapy Research, 23, 86-104.

*Nissen-Lie, H. A., Ronnestad, M. H., Hoglend, P. A., Havik, O. E., Solbakken, O. A., Stiles, T. C., \& Monsen, J. T. (2017). Love yourself as a person, doubt yourself as a therapist? Clinical Psychology \& Psychotherapy, 24, 48-60.

*Odyniec, P., Probst, T., Margraf, J., \& Willutzki, U. (2017). Psychotherapist trainees' professional self-doubt and negative personal reaction: Changes during cognitive behavioral therapy and association with patient progress. Psychotherapy Research, 27, $1-15$.

Orlinsky, D. E., Strauss, B., Ronnestad, M. H., Hill, C., Castonguay, L., Willutzki, U., . . Carlsson, J. (2015). A collaborative study of development in psychotherapy trainees. Psychotherapy Bulletin, 50, 21-4.

Orlinsky, D. E., \& Howard, K. I. (1987). A generic model of psychotherapy. Journal of Integrative \& Eclectic Psychotherapy, 6, 6-27.

Orlinsky, D. E., Rønnestad, M. H., \& Willutzki, U. (2004). Fifty years of psychotherapy process-outcome research: Continuity and change. In M. J. Lambert (Ed.), Bergin and Garfield's handbook of psychotherapy and behavior change (5th ed.) (pp. 307-393). New York: Wiley.

Orlinsky, D. E., \& Ronnestad, M. H. (2005). How psychotherapists develop: A study of therapeutic work and professional growth. Washington, DC: American Psychological Association. 
Owen, J., Tao, K. W., Drinane, J. M., Hook, J., Davis, D. E., \& Kune, N. F. (2016). Client perceptions of therapists' multicultural orientation: Cultural (missed) opportunities and cultural humility. Professional Psychology: Research and Practice, 47, 30-37.

*Pereira, J., Barkham, M., Kellett, S., \& Saxon, D. (2017). The role of practitioner resilience and mindfulness in effective practice: A practice-based feasibility study. Administration and Policy in Mental Health and Mental Health Services Research, 44, 691-704.

Raudenbush, S. W., \& Bryk, A. S. (2002). Hierarchical linear models (2nd ed.). Thousand Oaks: Sage.

*Rieck, T., \& Callahan, J. L. (2013). Emotional intelligence and psychotherapy outcomes in the training clinic. Training and Education in Professional Psychology, 7, 42-52.

Rønnestad, M. H., \& Skovholt, T. M. (2012). The developing practitioner: Growth and stagnation of therapists and counselors. New York: Routledge.

*Ryan, A., Safran, J. D., Doran, J. M., \& Muran, J. C. (2012). Therapist mindfulness, alliance and treatment outcome. Psychotherapy Research, 22, 289-297.

Sandell, R., Blomberg, J., Lazar, A., Carlsson, J., Broberg, J., \& Schubert, J. (2000). Varieties of long-term outcome among patients in psychoanalysis and long-term psychotherapy: A review of findings in the Stockholm Outcome of Psychoanalysis and Psychotherapy Project (STOPP). International Journal of Psychoanalysis, 81, 921-942.

*Sandell, R., Lazar, A., Grant, J., Carlsson, J., Schubert, J., \& Broberg, J. (2006). Therapist attitudes and patient outcomes. III. A latent class analysis of therapists. Psychology and Psychotherapy: Theory, Research and Practice, 79, 629-647. 
*Sandell, R., Lazar, A., Grant, J., Carlsson, J., Schubert, J., \& Broberg, J. (2007). Therapist attitudes and patient outcomes: II. Therapist attitudes influence change during treatment. Psychotherapy Research, 17, 196-204.

*Schauenburg, H., Buchheim, A., Beckh, K., Nolte, T., Brenk-Franz, K., Leichsenring, F., . . . Dinger, U. (2010). The influence of psychodynamically oriented therapists' attachment representations on outcome and alliance in inpatient psychotherapy. Psychotherapy Research, 20, 193-202.

*Schöttke, H., Flückiger, C., Goldberg, S. B., Eversmann, J., \& Lange, J. (2017). Predicting psychotherapy outcome based on therapist interpersonal skills: A five-year longitudinal study of a therapist assessment protocol. Psychotherapy Research, 27, 642-652.

*Stanley, S., Reitzel, L. R., Wingate, L. R., Cukrowicz, K. C., Lima, E. N., \& Joiner, T. E. J. (2006). Mindfulness: A primrose path for therapists using manualized treatments? Journal of Cognitive Psychotherapy, 20, 327-335.

Stiles, W. B. (2009). Responsiveness as an obstacle for psychotherapy outcome research: It's worse than you think. Clinical Psychology: Science and Practice, 16, 86-91.

*Swift, J. K., Derthick, A. O., Tompkins, K. A. (2018). The relationship between trainee therapists' and clients' initial expectations and actual treatment duration and outcomes. Practice Innovations, 3, 84-93.

*Toska, G. A., Neimeyer, G. J., Taylor, J. M., Kavas, A. B., \& Rice, K. G. (2010). Epistemology and allegiance: Exploring the role of therapists' epistemic commitments on psychotherapy outcomes. European Journal of Psychotherapy and Counselling, 12, $65-75$. 
Wampold, B. E., Baldwin, S. A., Grosse Holtforth, M., \& Imel, Z. (2017). What characterizes effective therapists. In L. G. Castonguay, \& C. E. Hill (Eds.), How and why are some therapists better than others: Understanding therapist effects. (pp. 37-53). Washington, DC: American Psychological Association.

Wampold, B. E., \& Imel, Z. E. (2015). The great psychotherapy debate: The evidence for what makes psychotherapy work. (2nd ed.). New York: Routledge.

Wampold, B. E., \& Brown, G. S. (2005). Estimating variability in outcomes attributable to therapists: A naturalistic study of outcomes in managed care. Journal of Consulting and Clinical Psychology, 73, 914-923.

Webb, C. A., DeRubeis, R. J., \& Barber, J. P. (2010). Therapist adherence/competence and treatment outcome: A meta-analytic review. Journal of Consulting and Clinical Psychology, 78, 200-211.

Wolff, M., \& Hayes, J. (2009). Therapist variables: Predictors of process in the treatment of alcohol and other drug problems. Alcoholism Treatment Quarterly, 27, 51-65. 


\section{Appendix A.}

The following terms were used as descriptors: (psychotherapy OR counseling OR counselling) AND (efficacy* OR outcome* OR effectiveness* OR therapist effect) AND (therapist characteristics OR counselor characteristics OR counsellor characteristics) OR (therapist* OR counselor* OR counsellor* OR analyst* OR psychoanalyst* OR clinician*) NEAR/3 (characteristic* OR qualit* OR variable* OR factor* OR feature* OR trait* OR propert*).

\section{Appendix B.}

For inclusion in the review, studies had to meet the following criteria:

1. Published 2000 or after

2. Original peer-reviewed quantitative studies

3. Study design: randomized trial or cohort study (longitudinal prospective design)

4. Predictor: therapists' observed or inferred pre-treatment characteristic ${ }^{1}$

5. Outcome of psychotherapy: a direct measure of patient outcome ${ }^{2}$

6. Psychotherapy: at least one of the treatments studied in the article is psychotherapy

7. Strength of association or significance is reported

8. Number of patients in smallest study group: $\geq 10$

9. Patient type: outpatients ${ }^{3}$

10. Number of therapists: $\geq 5$

11. Therapist training: fully trained or graduate-level therapists (i.e., working towards a doctoral degree)

\footnotetext{
${ }^{1}$ Excluding therapist-patient similarity or matching on discrete dimensions or variables.

${ }^{2}$ Excluding, e.g., patient drop-out.

${ }^{3}$ Two studies from Germany - where inpatient treatment is commonly used for depressive and anxiety disorders - and conducted by Dinger et al. (2007) and Schauenburg et al. (2010) were considered sufficiently similar in their populations to justify inclusion in the review.
} 


\section{Figure captions:}

Figure 1. Flow diagram of systematic search.

Table 1

Conceptual Schema of Therapist Characteristics Studied as Predictors of Outcome

\section{Task-instrumental}

Therapeutic attitudes and values

Professional self-efficacy

Professional self-doubt, difficulties

Coping strategies, countertransference management

\section{Intrapersonal}

Self- and other representations (e.g., introject, attachment styles) Intrapersonal traits (e.g., neuroticism)

Intrapersonal resources (e.g., mindfulness, resilience, emotional intelligence, wellbeing, reflective functioning) $\underline{\text { Personal characteristics }}$

Professional characteristics

Socio-emotional

Relational capacities (e.g., warmth, empathy, persuasiveness)

Relational manner

Feelings in therapy work

\section{Interpersona}

Interpersonal problems

Relational manner in private life

Social ability and attitudes in personal life 